\title{
Kernos
}

Revue internationale et pluridisciplinaire de religion grecque antique

$28 \mid 2015$

Varia

\section{Housing the Chosen. The Architectural Context of Mystery Groups and Religious Associations in the Ancient World}

\section{Anne-Françoise Jaccottet}

\section{(2) OpenEdition}

1 Journals

\section{Édition électronique}

URL : http://journals.openedition.org/kernos/2355

DOI : $10.4000 /$ kernos. 2355

ISSN : 2034-7871

Éditeur

Centre international d'étude de la religion grecque antique

\section{Édition imprimée}

Date de publication : 1 octobre 2015

Pagination : 285-289

ISBN : 978-2-87562-055-2

ISSN : 0776-3824

\section{Référence électronique}

Anne-Françoise Jaccottet, « Housing the Chosen. The Architectural Context of Mystery Groups and Religious Associations in the Ancient World », Kernos [En ligne], 28 | 2015, mis en ligne le 01 octobre 2015, consulté le 24 septembre 2020. URL : http://journals.openedition.org/kernos/2355 ; DOI : https://doi.org/10.4000/kernos.2355

Ce document a été généré automatiquement le 24 septembre 2020.

Kernos 


\title{
Housing the Chosen. The Architectural Context of Mystery Groups and Religious Associations in the Ancient World
}

\author{
Anne-Françoise Jaccottet
}

\section{RÉFÉRENCE}

NIELSEN Inge, Housing the Chosen. The Architectural Context of Mystery Groups and Religious Associations in the Ancient World, Turnhout, Brepols, 2014.1 vol. $21,5 \times 30 \mathrm{~cm} .322 \mathrm{p}$. (Contextualizing the Sacred, 2) ISBN : 978-2-503-54437-3.

1 Prendre et comprendre les mystères - et plus généralement les associations cultuelles - par l'architecture et le cadre spatial construit de leurs réunions est une perspective qu'on ne peut que saluer, en ce qu'elle se propose de combler un manque (relatif) dans la bibliographie existante et de "jeter une lumière plus 'objective' sur les mystères antiques et les associations religieuses $»^{1}$. Dans la ligne logique des travaux de l'auteur, l'ouvrage s'inscrit également, par sa thématique, dans un courant d'analyse du sacré par son cadre, qui se développe depuis une vingtaine d'années et concerne plus largement, outre l'Antiquité, toutes les périodes et les aires cultu(r)elles. C'est sans surprise que la première partie de l'ouvrage, qui est aussi et de loin la plus importante (187 pages sur 237 et 132 illustrations sur 136), s'attache à l'Architecture ${ }^{2}$, séparée en deux volets, un premier, d'une vingtaine de pages, consacré à la période préhellénistique, le second rassemblant le matériel des périodes hellénistique et romaine, et représentant le cœur même de l'étude. Si la troisième et dernière partie de l'ouvrage s'occupant de "Considérations de typologie ${ }^{3}$ est attendue et propose, en une douzaine de pages et sous forme de tableau commenté, une synthèse des analyses formelles qu'a permises le catalogue de la première partie, on est plus surpris par la présence d'une seconde partie dévolue aux «Fonctions cultuelles des groupes religieux»4. Divisée en 
deux chapitres - «Les rites d'initiation et la célébration des mystères $»^{5}$ (34 pages) et "Les assemblées religieuses et les banquets communs ${ }^{6}(7$ pages $)$ - cette section intermédiaire de l'étude se propose de dresser une histoire du développement des différents mystères (Éleusis, Samothrace, Lemnos, Thèbes, Dionysos, Isis, Cybèle, Mithra, $\mathrm{Christ}^{7}$ ) en dégageant les rites initiatiques et la pratique rituelle des mystères qui les caractérisent ; puis, selon le même principe, d'analyser les modes de réunion des diverses "associations religieuses", catégorie qui permet à l'auteur d'englober également les groupes gravitant autour des divinités syro-phéniciennes, de la « religion juive de la diaspora » et "des agape et autres services de l'église chrétienne ». La présence de cette deuxième partie, dont l'ambition est malheureusement inversement proportionnelle à la profondeur et à la précision de l'analyse, permet à l'auteur de présenter la synthèse conclusive sur les "Considérations de typologie " comme une combinaison du catalogue architectural de la première partie et des rites divers évoqués dans la deuxième partie, pour proposer une lecture fonctionnelle des locaux pris en compte qui sont répartis en trois types de formes : 1) The Temple-Type (Main Room and 'Holy of Holies') ; 2) The Cave/Grotto-Type ; 3) The Banqueting/House-Type. Les deux dernières catégories représentent, selon l'auteur, les solutions trouvées par les groupes qui se réunissaient en dehors des sanctuaires ou n'étaient pas acceptés par l'état et qui se réfugiaient donc dans des maisons privées, dans des grottes ou locaux souterrains ${ }^{8}$.

2 En fait de "lumière plus 'objective' sur les mystères et les associations religieuses " (p.2), l'étude d'Inge Nielsen s'embourbe dans une chaîne de préjugés très souvent désuets et dans une rationalité contre-productive par sa rigidité qui pervertissent fort malencontreusement la belle intention de départ. S'il peut être satisfaisant formellement - et peut-être rassurant - d'ordonner la réalité en catégories qui s'opposent les unes aux autres, reste le risque, non négligeable, d'enfermer le raisonnement dans des cases préétablies, d'y contraindre la matière documentaire et d'utiliser cette base, artificielle, pour édifier le raisonnement et tirer des conclusions. C'est ainsi qu'on se méfiera de l'usage systématique et fondamental des paires opposées telles qu'assemblées «à l'intérieur » ou "à l'extérieur » des sanctuaires, du « privé » et $\mathrm{du}$ "public », des associations "religieuses » et «non religieuses», des " old Greek Mysteries » face aux « religions orientales » et autres "rites ésotériques »; catégories qui posent d'autant plus problème qu'elles ne sont jamais définies et, ce qui est plus gênant, qu'elles ont été fortement remises en question dans leur pertinence en tant qu'outils heuristiques ces vingt dernières années.

Posée en axiome au seuil de l'enquête, la distinction entre initiation et mystères est la plus intéressante et mériterait d'être argumentée et véritablement expliquée, sur la base de définitions, ce qu'elle n'est malheureusement nulle part. On se contentera donc $\mathrm{du}$ principe que «les mystères sont une interprétation et une explication de l'initiation ", alors que "l'initiation peut être vue comme un test nécessaire avant d'être capable de participer aux mystères " $^{10}$; "ainsi des initiations peuvent être pratiquées individuellement séparément et des mystères peuvent être célébrés par des groupes d'initiés sans initiation $»^{11}$. Cette distinction s'articule sans doute à la reprise de la constatation que les mystères pratiqués dans le cadre d'associations, qui connaissent des réunions régulières rassemblant les mêmes membres, sont des célébrations qui se répètent et qui dès lors prolongent et donnent son sens à l'initiation, ponctuelle et unique. Mais ce point de départ, que l'on ne peut que 
soutenir, sert de tremplin à une séparation par trop schématique, voire dogmatique, entre deux catégories de mystères qui sont distinguées aussi bien par la chronologie et l'ancrage territorial que par l'articulation de la phase respectivement initiatique et mystérique des rites envisagés et de l'investissement personnel des initiés. Il y a les mystères collectifs, qui sont pré-hellénistiques, associés à un lieu et un sanctuaire précis et pour lesquels l'initiation était l'événement principal et dont les mystères était célébrés en lien (temporel et rituel) avec l'initiation; ce sont ce que l'auteur nomme "the old Greek collective mysteries » et dont Éleusis, Samothrace, Lemnos et Thèbes forment les modèles ${ }^{12}$. Bien distincts de ces formes véritablement grecques - qui subissent dès l'époque hellénistique "des mélanges avec d'autres rituels ésotériques d'autres régions $»^{13}-$ les mystères hellénistiques et romains sont individuels et associés en premier lieu à leurs dieux, tels ceux d'Isis, Cybèle, Mithra (des 'divinités orientales') et Dionysos ; si les deux premiers sont liés au départ à des sanctuaires, les deux derniers sont libres de tout lien territorial et fonctionnel et peuvent être pratiqués n'importe où dans des salles prévues spécialement à cet effet. C'est la privatisation et l'individualisation en marche dès l'époque hellénistique qui sont à l'origine du développement des mystères individuels, marque typique d'une importation de l'Orient ${ }^{14}$. Et c'est d'ailleurs dans ces mystères individuels pour lesquels le lien avec le dieu est au centre que la cérémonie d'initiation constituait le début d'une nouvelle vie (Dionysos, Mithra, chrétiens).

4 À ces présupposés s'ajoute une vision de l'initiation basée sur les rites de passage de Van Gennep - et assumée comme telle -, et de là l'assurance que les rituels d'initiation sont universels ${ }^{15}$. De cette universalité des rites découle l'universalité des types de locaux qui les ont abrités : "Since the function of these religious associations was often closely related to or indistinguishable from the 'individual' mysteries, the rooms in which they were performed might have also been of the same type, since form follows function» (p.3). Et cette universalité se construit sur la base des présupposés liés à l'idée d'initiation: "The atmosphere might have in fact been enhanced in the setting of a small dark room such as a crypt, a cave, or an adyton » (p. 4) ; quant aux mystères qui en sont la suite et la prolongation, s'ils ne sont pas liés à un sanctuaire, ils « devaient être tenus dans un bâtiment fermé pour assurer le secret des rites » (p. 4).

On comprend dès lors que de telles bases théoriques et méthodologiques influencent lourdement l'établissement du corpus des lieux de réunion et de rituel qui se voulait plus 'objectif' que les données textuelles ou iconographiques ${ }^{16}$. Et l'on comprend aussi que la deuxième partie, consacrée aux "fonctions cultuelles des groupes religieux ", dont la présence nous avait paru incongrue, est bien indissociable de la démarche présentée car c'est sur cette base fonctionnelle et rituelle que sont déterminés les espaces que l'auteur va chercher dans les vestiges, pervertissant par là même la quête archéologique qui devrait s'entendre sans préjugés fonctionnels. Mais «form follows function " (p. 4). Cet axiome - encore un - mériterait une discussion approfondie et un débat d'entrée de jeu, alors qu'il n'apparaît dans l'ouvrage que tacitement, comme à l'insu du plein gré de l'auteur, par les multiples concessions qu'elle doit faire pour englober les exemples concrets concernés dans les trois types fixés (Temple-Type, Cave/Grotto-Type, Banqueting/House-Type). L'architecture est-elle véritablement fonctionnelle? Comment concilier cette assertion avec le constat constant dans l'ouvrage, et parfaitement vérifié par ailleurs, que les espaces sont la plupart du temps multifonctionnels? Comment comprendre, sur cette base fonctionnelle, que l'on trouve dans des associations décrétées "non religieuses » les mêmes éléments spatiaux et 
architecturaux que dans les «associations religieuses »? Comment faire du telesterion d'Éleusis un temple? Par la forme? Par la fonction? Comment imaginer les rites de Samothrace avant la monumentalisation tardive du sanctuaire? Les initiations et les mystères ont-ils forcément besoin d'un cadre construit, retiré, abrité des regards non autorisés pour exister? Peut-on utiliser l'iconographie présente dans un espace construit pour en induire la fonction? Cette dernière question eût été nécessaire, pour prendre un seul exemple, avant de faire, en 13 lignes et sans discussion (p. 217), de la trop célèbre fresque de la mégalographie de Pompéi le témoignage («might reveal») de la tenue de drames mystériques, dans les lieux mêmes, avec en note 142, la précision suivante: « However, it is important to remember that the room with the paintings was not accessible to the public, but rather functioned as a meeting hall for the thiasos and thus did not reveal forbidden secrets for unauthorized persons ». Où sont donc les réflexions? Où trouver la prise de distance salutaire face aux modèles préétablis, et qui plus est largement dépassés?

On regrettera que cet ouvrage, au titre si prometteur, ait fait l'économie d'un questionnement de fond, d'une problématisation, d'un effort de définition et surtout d'une mise à jour des bases méthodologiques et théoriques. Cela en mine la crédibilité à la racine.

7 Reste alors à saluer le catalogue des lieux de culte et de réunion proposé dans la première partie et sa reprise sous forme de tableau synthétique dans la troisième. La mise à disposition des plans des différents sanctuaires, locaux ou espaces concernés, avec leurs données métriques dans le tableau, est un gain de temps considérable pour le chercheur. Mais il convient de mettre en garde l'utilisateur pressé. L'état de la documentation est malheureusement largement dépassé, lui aussi. La bibliographie, abondante pour les publications antérieures aux années 1990 (et trop largement focalisée sur les titres en allemand et anglais), ne tient pas compte des publications les plus récentes. Et si la date de signature de la préface (août 2012) nous fait comprendre qu'il est illusoire d'y chercher les ouvrages des trois ou quatre dernières années, elle n'explique pas les manques évidents de titres de référence publiés depuis la fin des années 1990. Ce manque est manifeste dans tous les domaines mais particulièrement dans la prise en compte des lieux de culte et de réunion chrétiens qui ont connu, comme on le sait, des découvertes et publications importantes ces dernières années.

Par l'état de la documentation comme dans les présupposés théoriques cet ouvrage n'est malheureusement pas à sa place dans les nouveautés 2014.

\section{NOTES}

1. "The spatial and architectural settings of assemblies have been mostly overlooked in the large corpus of recent scholarship that has studied the meaning of ancient mysteries and religious associations ", p. 1 ; « Architecture has been neglected in this discourse though I propose that it can throw a more 'objective' light on ancient mysteries and religious associations, and necessarly on the social and religious institutions that created them ", p. 2. 
2. Part I : The Architecture, p. 9-194.

3. Part III : Considerations of Typology, p. 241-253.

4. Part II : The Cultic Functions of Religious Groups, p. 196-237.

5. Chap. 5 : The Initiation Rites and the Celebration of Mysteries, p. 197-230.

6. Chap. 6 : Religious Assemblies and Communal Banquets, p. 231-237.

7. La liste des mystères pris en compte dans la première partie est plus large, incluant également les sanctuaires d'Andania, Délos, Lycosoura, Mantinée, Mégalopolis, Pergame, Priène, mais aussi des groupes comme les pythagoriciens, les orphiques, les syssities ou gene aristocratiques et toutes sortes d'associations dionysiaques, technites ou autres.

8. «Not all groups met in sanctuaries or were accepted by the state. In these groups, members either met in private houses [...], or in non-visible localities, like grottos and caves, natural or artificial », p. 250.

9. « Mysteries, which I define as an interpretation and explanation of the initiation... », p. 1.

10. "Initiations can be regarded as necessary test before being able to participate in the mysteries », p. 197.

11. "Thus initiations could be performed individually separately and mysteries could be celebrated by groups of initiates without initiations ", p. 196.

12. Toutes les références p. 2. Cf. aussi p. 197 sq.

13. "Nevertheless during the Hellenistic and Roman periods these rituals had become mixed with other esoteric rituals from other regions ", p. 2.

14. "With the development of the globalized and cosmopolitan cities of the Hellenistic and Roman periods, people had become increasingly privatized in their affairs, including participation in private mysteries and initiations that were not sanctioned by society. Such participation can be observed in the mysteries of Dionysos and Mithras as well as in early Christianity. Theses private or personnal mysteries - to contrast with collective mysteries were clearly adopted from the Orient where they were performed as priestly initiations ", p. 2 .

15. "These aspects of initiation rituals appear universal. As Van Gennep, The Rites of Passage, p. 92 », p. 4, n. 11.

16. On regrettera que ces bases théoriques et méthodologiques soient relayées, par le biais d'une version abrégée de l'ouvrage, dans le tout récent Companion to the Archaeology of Religion in the Ancient World, R. RAJA, J. RÜPKE (éd.), Chichester, 2015, sous le titre «Buildings of Religious Communities », p. 279-292.

\section{AUTEURS}

\section{ANNE-FRANÇOISE JACCOTTET}

Université de Genève 